\title{
The Efficacy and Safety of Tetrachlorodecaoxide in Comparison with Super-oxidised Solution in Wound Healing
}

\author{
Romil Parikh ${ }^{1}$, Girish Bakhshi ${ }^{1}$, Madhushree Naik ${ }^{1}$, Bhargav Gaikwad ${ }^{2}$, Kavita Jadhav ${ }^{1}$, \\ Mukund Tayade ${ }^{1}$ \\ Departments of ${ }^{1}$ Surgery and ${ }^{2}$ Department of Preventive and Social Medicine, Grant Government Medical College, Mumbai, India
}

Background Some of the relatively newer, more efficacious, and potent topical wound dressing solutions include tetrachlorodecaoxide and super-oxidised solution. This study compares the efficacy and safety of these two drugs.

Methods This is a block-randomised, double blind, parallel-arm, post-marketing study. One hundred fifty patients with ulcers (75 blocks uniform for sex, ulcer aetiology, diabetes mellitus, and wound area score) were randomised into the two treatment arms. Patients were observed for eight weeks with weekly assessments. One hundred and twenty patients completed the study. Wound healing was objectively assessed by measurement of wound area, scoring of wound exudation and tissue type, and using the pressure ulcer scale of healing Tool (validated for multiple wound aetiologies). Subjective improvement in pain was noted using a visual analogue scale. Both groups were compared using Mann-Whitney $\mathrm{U}$ test on all indicators.

Results Difference in change in wound tissue type in the two groups was significant ( $\alpha=$ 0.05 ) by intention-to-treat (IT) and per-protocol (PP) analysis at the end of week two (ITT and $P P, P<0.001)$ and week four (IT, $\mathrm{P}=0.010 ; \mathrm{PP}, \mathrm{P}=0.009)$. $\mathrm{P}$-values for other comparisons were not significant $(P>0.05)$. No study-related adverse events were observed.

Conclusions Both drugs are efficacious. Tetrachlorodecaoxide yields healthy granulation tissue earlier. Both drugs appear to be safe for application.

Keywords Tetrachlorodecaoxide / Superoxidized water / Ulcer / Wound healing
Correspondence: Romil Parikh Department of Surgery, Grant Government Medical College, Flat No. 401, Shiv-0m, Plot No. 150, 10th Road, Khar West, Mumbai-400052, Maharashtra, India

Tel: +91-976-949-4271

Fax: $+91-22-23735599$

E-mail: romil.r.parikh@gmail.com

Acknowledgement: Dr. TP Lahane, Dean, Grant Govt. Medical College, Mumbai, India for his motivation and support. Dr. Rajesh Tiwari for technical assistance. Short Term Studentship programme by Indian Council of Medical Research.

Source of fund: Short Term Research Grant Programme by Maharashtra University of Health Sciences, Nashik, India.

No potential conflict of interest relevant to this article was reported.

Received: 29 Jan $2016 \bullet$ Revised: 11 May $2016 \bullet$ Accepted: 31 May 2016

pISSN: 2234-6163 • elSSN: 2234-6171 • http://dx.doi.org/10.5999/aps.2016.43.5.395 • Arch Plast Surg 2016;43:395-401

\section{INTRODUCTION}

The science of wound management has developed rapidly in the past several years with the advent of new materials, techniques, and a deeper understanding of the biology of wound healing. Some of the relatively new, more efficacious, and potent topical wound dressing solutions include tetrachlorodecaoxide and super-oxidised solution (SOS) [1-4].

\section{Super-oxidised solution}

SOS is a safe and efficient wound care product [1] that moistens, lubricates, debrides, and reduces the microbial load of various types of lesions [5]. SOS is an electrochemically processed aqueous solution manufactured from pure solutions, which is 
rich in reactive oxygen species. It is a powerful anti-microbial. It is a stable, non-flammable, and noncorrosive bactericidal, virucidal, fungicidal, and sporicidal solution [5] that is ready to use with no further dilution or mixing.

\section{Tetrachlorodecaoxide}

Tetrachlorodecaoxide (TCDO) serves as a step in contribution to progress in wound healing through a newer concept based on the direct activation of the macrophage system, along with an increase in partial pressure of oxygen in the wound $[2,6]$. This is important in the healing of wounds, especially for those of a chronic character [7]. TCDO is an aqueous solution that moistens the wound. It contains bio-activated oxygen carrier. It breaks the vicious cycle of hypoxia in a wound. It contributes to meeting the increased oxygen demand involved in phagocytic activation adequately, without compromising the physiological degree of local hypoxia required for neo-angiogenesis [6]. The bactericidal action of TCDO has been demonstrated in vitro. In addition, TCDO also has mitogenic properties on fibroblasts and new blood vessels required for effective wound healing. Haemactivated decomposition of TCDO does not give rise to any toxic metabolite [8].

Both TCDO and SOS, individually, have been compared with other older alternatives in various studies $[3,4,9]$. This study is intended to directly compare the efficacy of these two drugs with each other.

The primary objective of this study is to evaluate the efficacy of TCDO in comparison to that of SOS in wound healing objectively, assessed by measurement of wound size, and also by a scoring system for wound exudation and wound tissue type.

The secondary objectives are to evaluate the efficacy of TCDO in comparison to that of SOS in subjective improvement of pain and discomfort felt by the patient, and to evaluate the safety and tolerability of the study treatments in terms of side effects and adverse effects, if any are observed over the study duration.

\section{METHODS}

This study is a double blind, comparative, parallel-arm, blockrandomised, post-marketing study. Patients were enrolled in blocks of two, to ensure uniformity within a block, as per ulcer aetiology, sex, diabetic status (diabetes mellitus-uncontrolled, controlled, and absent) and wound size score by the pressure ulcer scale of healing (PUSH) Tool. Using computerised randomization, patients in each block were randomly assigned to two treatment groups: A (TCDO) and B (SOS). Thus the allocation ratio remained 1:1. Patients and wound assessors (investigators in charge of data collection and data management) were blinded to treatment allocation. Wound dressers and the data analyser (the statistician writing the report) were not blinded. This study received ethical clearance and approval from the Institutional Ethics Committee.

Ethical approval: All procedures performed in the study involving human participants were in accordance with the ethical standards of the Institutional Ethics Committee and with the 1964 Declaration of Helsinki and its later amendments or comparable ethical standards.

Written informed consent was obtained from each participant enrolled in the study. The study sample included patients with ulcers of varied aetiologies. There was no discrimination on basis of caste, creed, or socio-economic status of the patient.

\section{Inclusion criteria}

Adult patients aged 18 years or older presenting with ulcers of varied aetiology, including pressure ulcers, venous ulcers, diabetic foot ulcers, post-traumatic wounds, postoperative complicated/non-healing wounds, amputation stumps, burn wounds, abscesses, and ulcers due to Fournier's gangrene.

\section{Exclusion criteria}

Pregnant or breast-feeding women, patients who are simultaneously participating in another clinical trial with an unlicensed drug or who have done so within a one-month period, patients not willing to participate in the study, patients with a known hypersensitivity to any of the drugs, and moribund patients.

This study was conducted in the department of surgery of a tertiary care hospital in Mumbai, India. The subjects were in-patients from six wards of the department. Patients were enrolled and assessed between August 2013 and October 2015.

A set of 463 patients were screened on a continuous basis (Fig. 1), out of which 150 patients met all criteria. Thus 75 blocks were enrolled. Within each block, the patients were randomly assigned to the treatment groups by computer-generated random assignment. A set of 15 patients were non-compliant with the wound-dressing protocol or wished to be discharged for home care. In such cases, the entire block (total $=15$ blocks, $\mathrm{n}=30$ ) was discarded from the study analysis, maintaining an allocation ratio of $1: 1$. Thus 60 blocks $(n=120)$ completed the trial.

Group A (TCDO) was treated with a solution of $100 \mathrm{~mL}$ that contained $1.037 \mathrm{mg}$ tetrachlorodecaoxide in an aqueous base. Group B (SOS) was treated with commonly used solution prepared as per patented Microcyn technology (Oculus Innovative Sciences, Petaluma, CA, USA). The agents were applied using disposable dark-tinged spray pump bottles. Wound dressing was done daily, twice a day, by first-year residents (PGY1) with test/ 


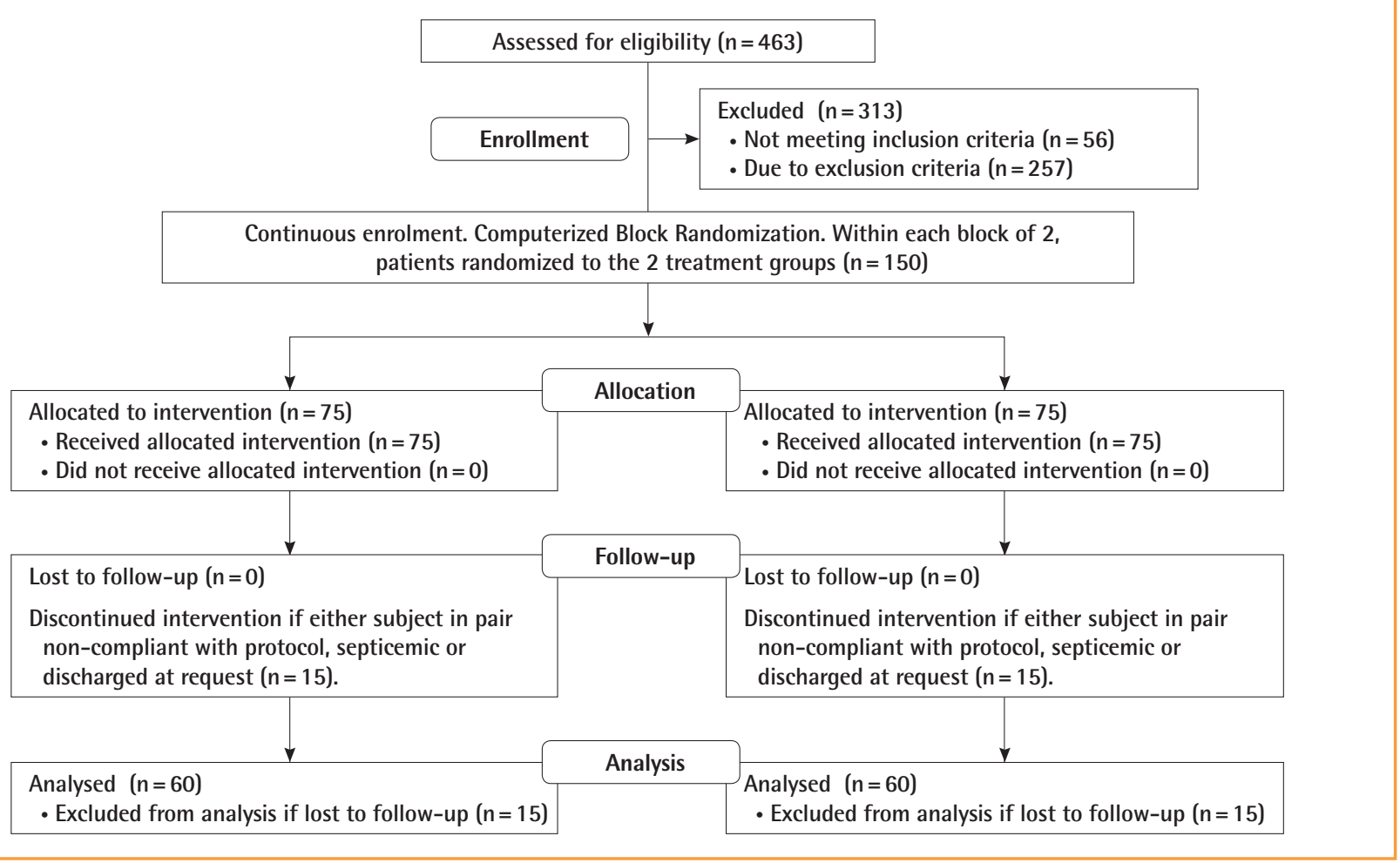

comparator agents, as per institutional protocol. After a 48-hour post-admission wash out period of standard care (scrubbing+ povidone dressing), the patients were assessed on day 0 (baseline), 1, 4, 7, 14, 2128,42 , and 56. On each assessment day, the following procedure was followed: (1) Step 1: Initial examination of the wound was done. The investigators assessed the wounds as per the protocol, including measurement of wound area, scoring of wound exudation and tissue type, and usage of the PUSH Tool. (2) Step 2: The wound was cleaned thoroughly and any necrotic tissue was debrided/ removed with forceps. (3) Step 3: Dressing was done with gauze after applying the test/comparator agent to the wound surface, so as to form a thin moist film of agent on the entire surface of the wound.

Wound recovery was assessed by decrease in wound size (approximate area), which was the primary efficacy variable, as well as improvement in scores of wound exudation and wound tissue type as per the PUSH Tool. Total recovery was measured by the total scores obtained using the PUSH Tool. The scores of both treatment groups were compared with each other for analysis of the comparative efficacies of the treatment agents. For wound area, the longest distances in length (centimetres) and width (centimetres) were measured and multiplied to give an approximate area of wound (square centimetres). In addition, this area was scored as per the PUSH Tool (ranging from 0 to 10). Wound exudation was scored according to its observed quantity (none, light, moderate, and heavy awarded 0, 1, 2, and 3 points respectively, as included in the PUSH Tool), quality (serous, sero-sanguinous, and purulent awarded 1, 2, and 3 points respectively), and odor (if present, awarded 1 point). Thus the range of total score for wound exudation is 0-7 points. Wound tissue type was scored as per the PUSH Tool (epithelial tissue, granulation tissue, slough, and necrotic tissue awarded 1 , 2,3 , and 4 points respectively). Additionally, total scores as given by the PUSH Tool on basis of wound area, observed quantity of wound exudation, and wound tissue type were computed. For subjective assessment of pain felt by the patient, the visual analogue scale (range, 0-10; 0 indicates least and 10 the maximum amount of pain) was used before the wound dressing was done. As per hospital standard of care protocols, all patients with infected ulcers were administered the antibiotic metronidazole. Wound culture sensitivity, complete blood check-up, renal and liver function tests, serum protein values, and blood sugar values were recorded for days $0,14,28$, and 56 only. After day 56 , the treatment was continued as per requirement; however, no assessments were included in the trial records.

Information on adverse events/side effects was to be obtained at each assessment visit (except the visit on day 0) as response to a non-leading question: "Have you had any new symptoms or increase in discomfort since your last assessment visit?" Additionally, any abnormal findings from physical examination or 
laboratory tests considered as adverse events/side effects by the investigator had to be documented as such.

\section{Statistical analysis}

There is no study comparing the efficacies of these drugs against each other. This was concluded after a literature search on PubMed and Medline using key words: "TCDO”, "Oxoferin”, "WF 10", "Immunokine", "Electrolysed water", "Superoxidised Solution” and "Oxum". Since this is the first study comparing the efficacy of these two drugs, there was no general data available to calculate the sample size statistically. However, on the basis of the primary hypothesis, the statistical power $(1-\beta)$ calculated after hypothesis testing is 0.81 . In this study, the two-tailed hypothesis is that there is a difference in the efficacies of the two treatment options. Therefore, the null hypothesis is that the two drugs are equally efficacious.

Data was analysed using the software GraphPad Prism Version 6.03 (C) 1992-2013 GraphPad Software, Inc. All Rights Reserved). The significance level ( $\alpha$ ) was set at 0.05 in this study. Normality of the sample was tested using the Kolmogorov-Smirnov goodness of fit test. Pearson's chi-square test was applied to qualitative variables with binary outcomes. For quantitative variables, as data was discrete, a non-parametric test was applied. To compare the two groups, by multiple parameters, the Mann-Whitney $U$ test was applied on each.

Due to attrition, intention-to-treat (ITT) analysis was performed on all patients enrolled $(n=150)$. For this purpose, the missing variables were estimated using a last observation carried forward approach. Additionally, per-protocol (PP) analysis was performed on all patients who completed the trial successfully $(\mathrm{n}=120)[10]$.

\section{RESULTS}

Recruitment was as shown in the CONSORT 2010 Participant Flow Diagram (Fig. 1). The study population included patients with ulcers of varied aetiology (Table 1).

Several population traits, as recorded on day 0 (baseline-first assessment), were analysed and compared between the two groups. The demographics of the population in both groups were similar. The mean age was 46.7 years (standard deviation, \pm 12.39 ). The significance level was set at 0.05 . The difference in the two groups was not significant $(P=0.608)$. The pattern of body mass index (BMI) of the total population $(n=150)$ followed normal distribution. The mean BMI was 22.55 (standard deviation \pm 3.47$)$. Comparison of the $\mathrm{BMI}$ in both treatment groups $(\mathrm{P}=0.469)$ showed similar distribution. Males formed $88 \%$ of the study population. Bacterial colonization was present in all enrolled cases. Difference in hemoglobin count $(\mathrm{P}=0.925)$, total leucocyte count $(\mathrm{P}=0.357)$, serum levels of sodium $(\mathrm{P}=$ $0.354)$, potassium $(\mathrm{P}=0.356)$, creatinine $(\mathrm{P}=0.918)$, urea $(\mathrm{P}=$ $0.219)$, albumin $(\mathrm{P}=0.711)$, globulin $(\mathrm{P}=0.430)$, total protein $(\mathrm{P}=0.572)$, alkaline phosphatase $(\mathrm{P}=0.727)$, total bilirubin $(\mathrm{P}=0.118)$, aspartate transaminase $(\mathrm{P}=0.636)$, and alanine transaminase $(\mathrm{P}=0.452)$ were all statistically non-significant amongst the two treatment groups $(\mathrm{P}>0.05)$. Four patients were hypertensive. Only two blocks had hypertensive patients.

The attrition rate was $20 \%(\mathrm{n}=30,15$ blocks). Due to this limitation, ITT analysis was performed along with PP analysis.

Significant wound healing occurred in each group. Freidman's repeated measures analysis of variance was applied to compare change in all indicators in both Group A and B, all of which

\begin{tabular}{|c|c|c|c|c|c|}
\hline \multirow[b]{2}{*}{ Etiology } & \multicolumn{2}{|c|}{ Group A (TCDO) } & \multicolumn{2}{|c|}{ Group B (SOS) } & \multirow[b]{2}{*}{ Total } \\
\hline & Diabetic & $\begin{array}{c}\text { Non- } \\
\text { diabetic }\end{array}$ & Diabetic & $\begin{array}{l}\text { Non- } \\
\text { diabetic }\end{array}$ & \\
\hline Abscess & 6 & 8 & 6 & 8 & 28 \\
\hline Amputation stump & 10 & 2 & 10 & 2 & 24 \\
\hline Bedsore & 0 & 5 & 0 & 5 & 10 \\
\hline Diabetic foot ulcer & 10 & - & 10 & - & 20 \\
\hline Fournier's gangrene & 0 & 2 & 0 & 2 & 4 \\
\hline Surgical wound gape & 0 & 1 & 0 & 1 & 2 \\
\hline Traumatic ulcer & 0 & 17 & 0 & 17 & 34 \\
\hline Venous ulcer & 0 & 14 & 0 & 14 & 28 \\
\hline Total & \multicolumn{2}{|c|}{75} & \multicolumn{2}{|c|}{75} & 150 \\
\hline
\end{tabular}

Table 2. Total change in indicators (P-values for Mann-Whitney U test: $\alpha, 0.05 ; 1-\beta=0.81$ )

\begin{tabular}{|c|c|c|c|c|c|c|c|c|c|c|c|c|}
\hline & \multicolumn{2}{|c|}{ Day 0} & \multicolumn{2}{|c|}{ Day 07} & \multicolumn{2}{|c|}{ Day 14} & \multicolumn{2}{|c|}{ Day 28} & \multicolumn{2}{|c|}{ Day 42} & \multicolumn{2}{|c|}{ Day 56} \\
\hline & $\mathrm{ITT}$ & PP & $\mathrm{ITT}$ & PP & $\mathrm{ITT}$ & PP & $\mathrm{ITT}$ & PP & $\mathrm{ITT}$ & $\mathrm{PP}$ & ITT & PP \\
\hline Wound area difference & 0.47 & 0.46 & 0.51 & 0.49 & 0.63 & 0.51 & 0.67 & 0.59 & 0.66 & 0.60 & 0.63 & 0.58 \\
\hline Wound exudation difference & 0.38 & 0.35 & 0.84 & 0.72 & 0.84 & 0.84 & 0.65 & 0.74 & 0.59 & 0.53 & 0.32 & 0.36 \\
\hline Wound tissue type difference & 0.94 & 0.86 & 0.62 & 0.84 & $<\underline{0.001}$ & $<\underline{0.001}$ & $\underline{0.010}$ & $\underline{0.009}$ & 0.37 & 0.28 & 0.58 & 0.46 \\
\hline PUSH Score difference & 0.98 & 0.97 & 0.61 & 0.54 & $\overline{0.63}$ & $\overline{0.62}$ & 0.14 & 0.11 & 0.36 & 0.36 & 0.36 & 0.76 \\
\hline VAS score difference & 0.57 & 0.54 & 0.71 & 0.63 & 0.39 & 0.31 & 0.45 & 0.40 & 0.26 & 0.24 & 0.19 & 0.17 \\
\hline
\end{tabular}


Table 3. Median score (with range): Group A-TCDO vs. Group B-SOS

\begin{tabular}{|c|c|c|c|c|c|c|}
\hline & Day 0 & Day 7 & Day 14 & Day 28 & Day 42 & Day 56 \\
\hline \multicolumn{7}{|c|}{ Wound area score } \\
\hline TCDO & $10(3-10)$ & $10(3-10)$ & $10(2-10)$ & $10(2-10)$ & $10(0-10)$ & $10(0-10)$ \\
\hline SOS & $10(3-10)$ & $10(3-10)$ & $10(3-10)$ & $10(3-10)$ & $10(2-10)$ & $10(0-10)$ \\
\hline \multicolumn{7}{|c|}{ Exudation score } \\
\hline TCDO & $7(3-7)$ & $6(3-7)$ & $4(2-6)$ & $3(2-5)$ & $2(0-5)$ & $2(0-5)$ \\
\hline SOS & $7(4-7)$ & $5(3-7)$ & $4(2-7)$ & $4(2-6)$ & $3(0-5)$ & $2(0-4)$ \\
\hline \multicolumn{7}{|c|}{ Tissue type score } \\
\hline TCDO & $4(3-4)$ & $3(2-4)$ & $2(2-3)$ & $2(1-3)$ & $2(1-3)$ & $1(1-2)$ \\
\hline SOS & $4(3-4)$ & $3(3-4)$ & $3(3-4)$ & $3(2-3)$ & $2(2-3)$ & $2(1-2)$ \\
\hline \multicolumn{7}{|c|}{ PUSH score } \\
\hline TCDO & $16(9-17)$ & $16(7-17)$ & $15(5-17)$ & $13(5-16)$ & $13(1-16)$ & $12(1-15)$ \\
\hline SOS & $16(9-17)$ & $16(7-17)$ & $15(6-17)$ & $14(6-16)$ & $13(4-15)$ & $12(1-14)$ \\
\hline \multicolumn{7}{|l|}{ VAS score } \\
\hline TCDO & $8(0-10)$ & $8(0-10)$ & $7(0-10)$ & $6(0-9)$ & $5(0-8)$ & $5(0-8)$ \\
\hline SOS & $8(2-10)$ & $8(2-10)$ & $7(0-10)$ & $7(0-9)$ & $6(0-9)$ & $6(0-9)$ \\
\hline
\end{tabular}

\section{Fig. 2. Wound tissue type-Day 14}

TCDO, tetrachlorodecaoxide; SOS, super oxidized solution.

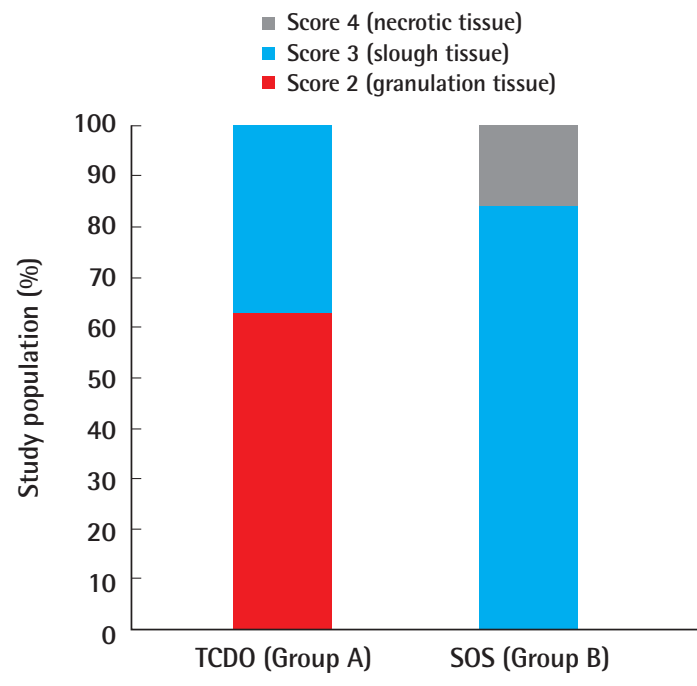

turned out to be highly significant (all $\mathrm{P}<0.001)$. The efficacies of TCDO and SOS were compared using a non-parametric Mann-Whitney U test. The test was performed on total change, from baseline, at the end of each week, in the following indicators: wound area, wound exudation, wound tissue type score, total PUSH scores, and VAS scores.

At the end of second and fourth week (day 14 and 28), the total difference in wound tissue type between the two treatment groups was statistically significant (Table 2 ), which was clinically supported by the median wound tissue type scores (Table 3 ). Thus Group A (tetrachlorodecaoxide), yielded granulation tissue earlier (Figs. 2, 3). Otherwise, the difference in the total change in all indicators on all assessment days was non-signifi-

\section{Fig. 3. Wound tissue type-Day 28}

TCDO, tetrachlorodecaoxide; SOS, super oxidized solution.

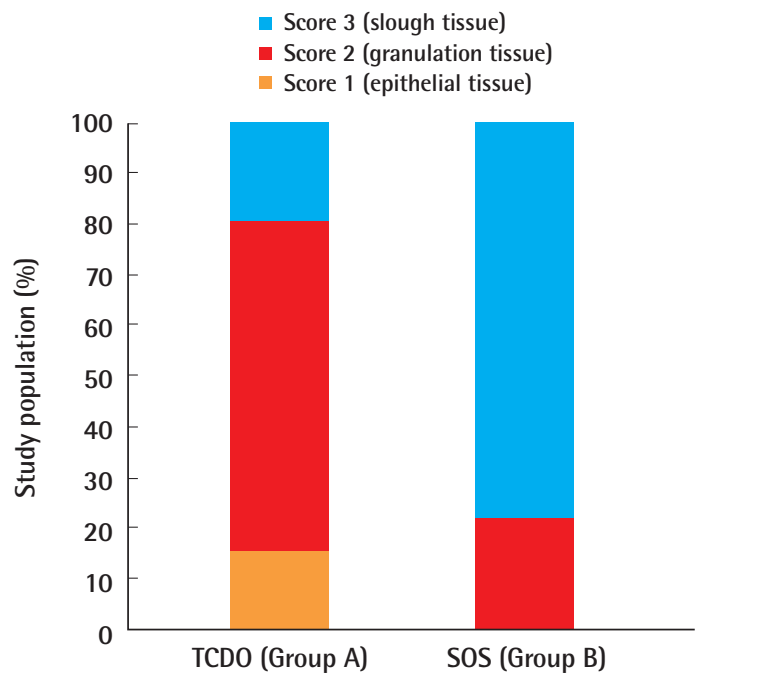

cant $(\mathrm{P}>0.05)$ (Table 2). No adverse events were observed. One patient in Group B developed septicaemia and was transferred out of the ward. That entire pair was dropped from the study per protocol as stated above.

\section{DISCUSSION}

This study compares the efficacy of two topical wound dressing agents: TCDO and SOS. Ulcers of various aetiologies were included in the study. To diminish the chances of a selection bias, patients enrolled were block-randomised, resulting in an allocation ratio of $1: 1$. Both groups have an equal number of cases per ulcer aetiology, wound area score, sex, and diabetic status (dia- 
betes mellitus). Due to feasibility issues owing to limited resources, out of the several factors that affect wound healing, only the four factors mentioned have been matched. Factors such as circulation at site could not be matched. Baseline differences in the two groups in terms of age, BMI, hypertension, pulse, and blood pressure are not significant, so the chances of selection bias are further reduced. Bacterial colonization of the wound was present in every case in both groups, but bias may arise from differences in bacterial load in each case. Wound tissue type and exudation scores are categorical comparators, and hence offer a weak comparison in this regard. Since the wound assessors in charge of data collection and management were blinded, chances of experimenter-expectancy bias were nullified.

To measure wound healing, this study employed the PUSH Tool (Version 3.0) [11,12]. This tool was primarily created for pressure ulcers, but it has been validated for ulcers with other aetiologies as well [13]. In a study by Pillen et al. [14], ten different instruments proposed to measure wound healing were evaluated. None were found to satisfy all criteria required for instrument validation, defined by content and criterion validity, intra- and inter-rater reliability and sensitivity to change. Among various available tools for wound assessment, the predictive validity of the PUSH Tool has been demonstrated consistently in multiple studies [14].

For the wound tissue type, reliance on the PUSH Tool alone may underestimate the results. The PUSH Tool recognises only four broad categories of this indicator compared to a detailed assessment with the Bates-Jensen Wound Assessment Tool [14].

The attrition rate was as high as $20 \%(\mathrm{n}=30,15$ blocks $)$. Due to this limitation, ITT analysis was performed along with PP analysis. There were two reasons for attrition. Either the patient was non-compliant with the protocol, or the patient requested discharge for home care.

Another limitation of the study is the short follow-up (eight weeks only) in the study. This also led to an absence of wound closure data. The short follow-up was chosen for practical purposes. Our study site was a government-funded tertiary care hospital in an urban area. Depending on factors like their socioeconomic status, patients often request discharge for home care as soon as they can resume daily activities with ease. Thus, a longer follow-up period would have resulted in an even greater attrition rate in this study. Also, there is no way to ascertain whether the dressing protocol has been exactly followed after discharge. But the PUSH Tool has been validated and, even over shorter periods, proven to be effective in assessing wound healing $[11,13,14]$.

TCDO is a safe and efficacious topical wound dressing agent. This is a well-established fact. The drug has no major document- ed side effects. In 1986, Hinz et al. [2] conducted a multi-centric, double blind randomised clinical trial on 217 patients, with $0.9 \%$ normal saline as control. The study demonstrated three therapeutic effects in "difficult wounds": wound cleansing was intensified, the formation of granulation tissue and epithelialization was promoted, and there was marked reduction in wound size. In comparison with normal saline, TCDO was proven to be more efficacious [2]. Another study by Zenker et al. [3] determined the superiority of the quality of granulation tissue on application of TCDO in comparison with povidone-iodine solution. Further, they found that the strength of antibacterial activity of TCDO is comparable to povidone-iodine solution. More recently, in 2011, Yingsakmongkol et al. [15] conducted a double blind, randomised, placebo-controlled trial to evaluate the effect of TCDO on diabetic foot ulcers. They concluded that TCDO statistically significantly reduced wound severity scores, infection and inflammation, and necrotic tissue; it also enhanced the formation of granulation tissue. In this study, TCDO (Group A) showed statistically significant (two-tailed P-value $<0.05$ ) changes in all five parameters of healing - namely, wound area, exudation, total PUSH scores and VAS scores. Thus TCDO contributed positively to wound healing.

SOS or electrolysed water, is a safe and efficacious topical wound dressing agent. This has been demonstrated amply. In 2006, Paola et al. [1] conducted an open-label, non-randomised trial comparing the effects of SOS with povidone-iodine solution in diabetic foot ulcers. In terms of healing time and antibacterial activity, they found SOS to be significantly superior to povidone-iodine solution. In 2008, Dharap et al. [16] conducted a study demonstrating the efficacy and safety of SOS on 30 cases of venous ulcers. They found SOS improved the clinical status of the patients, reduced signs of inflammation, and proved to be a potent anti-infective. In 2009, Abhyankar et al. [9] studied the efficacy and safety of SOS on 30 patients with chronic wounds. They concluded that SOS was effective, well tolerated and superior to povidone-iodine solution. In this study, SOS (Group B) showed statistically significant (two-tailed P-value $<0.05$ ) changes in all five parameters of healing - namely, wound area, exudation, total PUSH scores and VAS scores. Thus SOS contributed positively to wound healing.

In this study, the efficacies of TCDO \& SOS were compared using a non-parametric Mann-Whitney U test. At the end of the second and fourth weeks, the difference in wound tissue type was statistically significant. At the end of the second week, the median score in Group A was 2, that is, granulation tissue (range, 3-2), whereas in Group B it was 3, that is, slough (range, 4-3). At the end of the fourth week, the median score in Group A was 2, that is, granulation tissue (range, 3-1), whereas in Group 
$\mathrm{B}$ it was 3, that is, slough (range, 3-2). The P-values at the end of week two (ITT and PP, P < 0.001) and week four (ITT, P = 0.010; $\mathrm{PP}, \mathrm{P}=0.009$ ) were significant. Therefore, in this study, TCDO yielded healthy granulation tissue about two weeks before SOS.

A significant P-value was obtained with ITT analysis, as well as with PP analysis, at the end of weeks two and four. This is strong evidence supporting the significance of the difference in wound tissue type. As discussed above, there is scientific evidence that TCDO helps in the formation of superior quality granulation tissue and is a weak antimicrobial $[3,15]$. SOS is a powerful antimicrobial $[1,9]$. In each enrolled case, the wound was colonised by bacteria. This may be the reason why the significant difference in wound tissue type was observed no sooner than the end of the second week. Future trials on exclusively aseptic wounds may provide more evidence for this hypothesis.

The $\mathrm{P}$-values of remaining indicators are greater than 0.05 , that is, not significant. This implies that statistically, at an $\alpha=0.05$ level of significance, there is not enough evidence to prove that the two drugs are not equally efficacious. Clinically, the observable results (effects on wound healing) in both groups per indicator, overall, are similar in distribution and comparable in nature.

For successful extrapolation of the results, more trials need to be conducted. Future trials should be double blinded and multicentric. Individual trials on ulcers with each aetiology should provide more focused and substantial results leading to increased efficiency of wound management. For making a responsible decision in management of surface wounds, local cost-effectiveness of both drugs must also be compared.

In conclusion, both TCDO and SOS, individually, are efficacious and potent topical wound dressing agents. They contribute to wound healing by reducing healing time. TCDO yields healthy granulation tissue earlier than SOS. Both topical agents are safe to use.

\section{REFERENCES}

1. Paola LD, Brocco E, Senesi A, et al. Super-oxidized solution (SOS) therapy for infected diabetic foot ulcers. Wounds 2006; 18:262-70.

2. Hinz J, Hautzinger H, Stahl KW. Rationale for and results from a randomised, double-blind trial of tetrachlorodecaoxygen anion complex in wound healing. Lancet 1986;1:825-8.

3. Zenker W, Thiede A, Dommes M, et al. Effectiveness of tetrachlorodecaoxide (TCDO) in the treatment of complicat- ed disorders of wound healing: a controlled study: TCDO versus PVP-iodine complex. Chirurg 1986;57:334-9.

4. Kapur V, Marwaha AK. Evaluation of effect and comparison of superoxidised solution (oxum) v/s povidone iodine (betadine). Indian J Surg 2011;73:48-53.

5. Bryant R. Super-oxidized water kills bacteria; demonstrates potential for healing. Dermatol Times 2005;26.

6. Youngman RJ, Wagner GR, Kuhne FW, et al. Biochemical oxygen activation as the basis for the physiological action of tetrachlorodecaoxide (TCDO).Z Naturforsch C 1985;40:40914.

7. Hinz J, Kuhne FW, Stahl KW. Local tetrachlorodecaoxide treatment to improve oxygen supply to non-healing wounds. Lancet 1984;2:630.

8. Ullmann U, Kuhne FW. In vitro investigations on the antibacterial action and the influence on the phagocytic chemiluminescence of tetrachlorodecaoxide--a new, non-metallic oxygen complex. Infection 1985;13 Suppl 2:S236-40.

9. Abhyankar SV, Venkatesh V, Karnad S, et al. Efficacy and safety of oxum in treatment of chronic wounds.J Indian Med Assoc 2009; 107:904-6.

10. Gupta SK. Intention-to-treat concept: a review. Perspect Clin Res 2011;2:109-12.

11. Stotts NA, Rodeheaver GT, Thomas DR, et al. An instrument to measure healing in pressure ulcers: development and validation of the pressure ulcer scale for healing (PUSH). J Gerontol A Biol Sci Med Sci 2001;56:M795-9.

12. European Pressure Ulcer Advisory Panel; National Pressure Ulcer Advisory Panel. Pressure ulcer prevention and treatment: clinical practice guideline. Washington, DC: National Pressure Ulcer Advisory Panel; 2009.

13. Hon J, Lagden K, McLaren AM, et al. A prospective, multicenter study to validate use of the PUSH in patients with diabetic, venous, and pressure ulcers. Ostomy Wound Manage 2010;56:26-36.

14. Pillen H, Miller M, Thomas J, et al. Assessment of wound healing: validity, reliability and sensitivity of available instruments. Wound Pract Res 2009;17:208-17.

15. Yingsakmongkol N, Maraprygsavan P, Sukosit P. Effect of WF10 (immunokine) on diabetic foot ulcer therapy: a double-blind, randomized, placebo-controlled trial. J Foot Ankle Surg 2011;50:635-40.

16. Dharap SB, Ghag GS, Kulkarni KP, et al. Efficacy and safety of oxum in treatment of the venous ulcer. J Indian Med As$\operatorname{soc} 2008$; $106: 326,8-30$. 